\title{
On the kinematics of the neutron star low mass X-ray binary Cen X-4
}

\author{
J. I. González Hernández ${ }^{1}$, R. Rebolo ${ }^{1,2,3}$, J. Peñarrubia ${ }^{3}$, J. Casares ${ }^{1}$, and G. Israelian ${ }^{1}$ \\ 1 Instituto de Astrofísica de Canarias, 38205 La Laguna, Tenerife, Spain \\ e-mail: [jonay;rrl;jcv;gil]@iac.es \\ 2 Consejo Superior de Investigaciones Científicas, Spain \\ 3 Max-Planck Institut für Astronomie, Königstuhl, 17, 69117 Heidelberg, Germany \\ e-mail: jorpega@mpia-hd.mpg.de
}

Received 29 November 2004 / Accepted 8 February 2005

\begin{abstract}
We present the first determination of the proper motion of the neutron star low mass X-ray binary Cen X-4 measured from relative astrometry of the secondary star using optical images at different epochs. We determine the Galactic space velocity components of the system and find them to be significantly different from the mean values that characterize the kinematics of stars belonging to the halo, and the thin and the thick disc of the Galaxy. The high metallicity of the secondary star of the system rules out a halo origin and indicates that the system probably originated in the Galactic disc. A statistical analysis of the galactocentric motion revealed that this binary moves in a highly eccentric $(e \simeq 0.85 \pm 0.1)$ orbit with an inclination of $\simeq 110^{\circ}$ to the Galactic plane. The large Galactic space velocity components strongly support that a high natal kick as a result of a supernova explosion could have propelled the system into such an orbit from a birth place in the Galactic disc. The high Li abundance in the secondary, comparable to that of stars in star forming regions and young stellar clusters like the Pleiades, may suggest a relatively recent formation of the system. Following the orbit backwards in time, we found that the system could have been in the inner regions of the Galactic disc 100-200 Myr ago. The neutron star might have formed at that moment. However, we cannot rule out the possibility that the system formed at a much earlier time if a Li production mechanism exists in this LMXB.
\end{abstract}

Key words. stars: individual: Cen X-4 - X-rays: binaries - astrometry

\section{Introduction}

Cen X-4 (V822 Cen) was discovered by the Vela $5 B$ satellite during an X-ray outburst in 1969 (Conner et al. 1969). During the decay of a second outburst in 1979, a type I X-ray burst was observed, which indicated that the X-ray source is a neutron star (Matsuoka et al. 1980). This system is a prototype low mass X-ray binary (LMXB) consisting of a compact object of $M_{\mathrm{NS}}=0.5-2.1 M_{\odot}$ (Shahbaz et al. 1993) and a donor star with a mass in the range $0.04 M_{\odot}<M_{2}<0.58 M_{\odot}$ (Torres et al. 2002).

The high Galactic latitude $\left(b \sim 24^{\circ}\right)$ of the system coupled with its distance $(\sim 1.2 \pm 0.3 \mathrm{kpc}$, Chevalier et al. 1989) places the object at the Galactic height of $\sim 0.4-0.6 \mathrm{kpc}$ and might suggest that it belongs to the Galactic halo. However, its relatively large centre-of-mass radial velocity $(\gamma=191 \pm 1$ $\mathrm{km} \mathrm{s}^{-1}$, Casares et al. 2005, in preparation) opens the possibility that the system received a natal kick as a result of the supernova explosion that generated the compact object and it might come from the Galactic disc. Such kicks have been proposed to explain the large transverse motions of neutron stars on the plane of the sky (e.g. Lyne \& Lorimer 1994).

The kinematic properties of other similar systems at high Galactic latitude have been studied by Mirabel et al. (2001, XTE J1118+40) and Mirabel \& Rodrigues (2003, Sco X-1) by measuring their proper motions. These authors argued that XTE J1118+40 and Sco X-1 have kinematics consistent with the most ancient stars and globular clusters in the inner Galactic halo. However, according to the the galactocentric orbits of these objects, an origin in the bulge or disc of the Galaxy cannot be ruled out. In fact, Gualandris et al. (2005) have recently suggested that the black hole binary XTE J1118+40 could have formed in the Galactic disc $\sim 2-5$ Gyr ago.

The kinematic properties of Cen X-4 can help us to understand its origin. Here, we report the first determination of the proper motion of this system via relative astrometry of the secondary star using optical images. This, together with the radial velocity and the distance, allows us to determine the kinematic parameters and study the galactocentric orbit of this important LMXB. 


\section{Proper motion}

Accurate proper motion measurements of LMXBs have been obtained using very long baseline array (VLBA) or very long baseline interferometry (VLBI) techniques at radio wavelengths and/or from optical observations of these systems at HST or large telescopes. Unfortunately, there are no reported observations of Cen X-4 with either of these facilities. However, several campaigns have carried out observations of this target using optical ground-based telescopes. We selected two images among those with better pixel scales: a plate from the Second Epoch Survey ${ }^{1}$ of the Southern Sky observed in May 1994 in the Anglo-Australian Observatory (AAO) with the UK Schmidt Telescope with a pixel scale of 1 arcsec, and a second image with a pixel scale of 0.238 arcsec obtained in May 2002 with the Wide Field Imager at the European Southern Observatory (ESO), Observatorio La Silla, using the Cassegrain focus of the $2.2 \mathrm{~m}$ MPG/ESO telescope.

The proper motion was determined using several tasks within IRAF. First, we measured the pixel coordinates of 40 stars including the target, Cen X-4, in both images (with the task IMCENTROID). Second, the 1994 image was registered on the 2002 one, by applying the coordinate transformation (using the tasks GEOMAP and GEOXYTRANS) between both frames of reference objects. We selected as reference objects those stars whose transformation residuals were lower than $2 \sigma$ above the rms of the transformation (first criterion). Third, we measured the relative shifts of all the stars between their positions corresponding to the 1994 and 2002 images in the reference frame of the 2002 image. We then rejected those stars whose proper motions were higher than $3 \sigma$ above the standard deviation of the motions of all the stars (second criterion). The whole procedure was iterated until we could match the reference objects with both criteria unambiguously. We aligned (using the tasks CCMAP and CCTRAN) the 2002 frame in right ascension and declination with these reference objects using the coordinates given by $2 \mathrm{MASS}^{2}$. In order to correct for geometrical distorsions with GEOMAP and CCMAP, we selected general geometry transformations up to order 2 . These take into account rotation, pixel/celestial coordinate scale and shift between the reference frames of both images. Finally, we computed the relative displacements of our target and estimated the error from the average relative motions of the reference stars (see Fig. 1). The computed proper motion is $\mu_{\alpha}=11 \pm 10 \mathrm{mas} / \mathrm{yr}$ and $\mu_{\delta}=-56 \pm 10 \mathrm{mas} / \mathrm{yr}$, which leads to an overall yearly motion of $\mu=-57 \pm 12$ mas/yr along a position angle with respect to the North Celestial Pole of $100^{\circ} \pm 12^{\circ}$. At a distance $d$

\footnotetext{
${ }^{1}$ Plates from this survey, also called Second Palomar Sky Survey (POSSII), have been digitized and compressed by the ST ScI. The digitized images are copyright (c) 1993-2000 by the Anglo-Australian Observatory Board and are distributed herein by agreement. All Rights Reserved. Produced under Contract No. NAS5-2555 with the National Aeronautics and Space Administration.

2 The Two Micron All Sky Survey is a joint project of the University of Massachusetts and the Infrared Processing and Analysis Center/California Institute of Technology, funded by the National Aeronautics and Space Administration and the National Science Foundation.
}

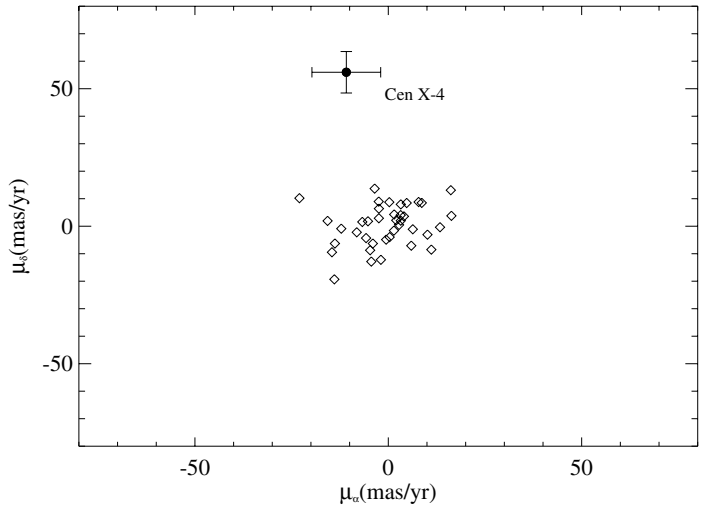

Fig. 1. Distribution of shifts in right ascension and declination for the 39 reference stars and the target (wide cross), Cen X-4. The error bars give the total error (see Sect. 2).

(in $\mathrm{kpc}$ ), this proper motion corresponds to a transverse velocity on the plane of the sky of $(270 \pm 56) d \mathrm{~km} \mathrm{~s}^{-1}$.

\section{Distance to Cen X-4}

In order to determine the space velocity of the system from the proper motion measurement we need to know its distance to the Sun. The optical properties of the secondary star can provide an estimate of the distance, although we found several discrepancies among the values obtained using different photometric filters. The mass range estimated for the secondary star of $0.04<M_{2} / M_{\odot}<0.58$ (Torres et al. 2002) leads to a stellar radius of $0.5<R_{2} / R_{\odot}<1.2$ by assuming that the secondary fills its Roche lobe. This radius, together with our spectroscopic estimate of the effective temperature, $T_{\text {eff }}=4500 \pm 100 \mathrm{~K}$ (González Hernández et al. 2005), provides an intrinsic bolometric luminosity of $0.095<L_{2} / L_{\odot}<0.371$. The apparent magnitude is $m_{V} \sim 18.2$ (van Paradijs et al. 1980) and the colour excess, $E_{B-V}=0.1 \pm 0.05$ (Blair et al. 1984). Using the bolometric corrections (Bessel et al. 1998) and the effective temperature, surface gravity and veiling from the accretion disc derived by González Hernández et al. (2005, submitted), we found $1.5<d_{m_{V}}(\mathrm{kpc})<3.6$. Note that Cen X-4 is highly variable in quiescence, with reported magnitudes $m_{\mathrm{V}} \sim$ 18-18.7 (Chevalier et al. 1989; Cowley et al. 1988). From the $m_{R} \sim 17.5$ and $m_{H} \sim 15.1$ (Shahbaz et al. 1993) and assuming $A_{R} / A_{V} \sim 0.83$ and $A_{H} / A_{V} \sim 0.21$ (Schaifers et al. 1982), we obtained $1.2<d_{m_{R}}(\mathrm{kpc})<2.9$ and $0.9<d_{m_{H}}(\mathrm{kpc})<2.3$. The distance obtained from the $V$ and $R$ filters in particular are rather uncertain because our veiling estimates and the assumed apparent magnitudes were determined at different epochs and are also affected by the contribution of a contaminant star (with $m_{V} \lesssim 20.5$ ) to the total flux (Casares et al. 2005, in preparation).

A more confident value of the distance can be calculated from the peak fluxes of the X-ray precursor observed in the 1969 outburst (Belian et al. 1972) and the X-ray burst detected during the 1979 outburst (Matsuoka et al. 1980). From the observed fluxes of $\sim 1.4 \times 10^{-6} \mathrm{erg} \mathrm{cm}^{-2} \mathrm{~s}^{-1}$ and $\sim 1 \times$ $10^{-6} \mathrm{erg} \mathrm{cm}^{-2} \mathrm{~s}^{-1}$ (for 1969 and 1979, respectively) and by assuming that they correspond to X-ray luminosities at the 
Eddington limit for a canonical neutron star $\left(L_{\mathrm{Edd}} \sim 1.6-2.7 \times\right.$ $10^{38} \mathrm{erg} \mathrm{s}^{-1}$ ), Chevalier et al. (1989) derived a distance of $1.2 \pm 0.3 \mathrm{kpc}$. This measurement can also be estimated by considering recent average peak luminosities of photospheric radius expansion bursts observed in globular clusters, which is (2-3.8) $\times 10^{38} \mathrm{erg} \mathrm{s}^{-1}$ (Jonker \& Nelemans 2004, and references therein). From these peak luminosities and the observed fluxes we obtained a distance of $d \simeq 1.4 \pm 0.3 \mathrm{kpc}$.

The space velocity calculated according to the derived proper motion exceeds the Galactic escape velocity (around $500 \mathrm{~km} \mathrm{~s}^{-1}$ in the solar neighbourhood) if the system is further than $1.7 \mathrm{kpc}$ from the Sun (see Sect. 4). Therefore, we assume hereafter a distance to Cen X-4 of $0.9<d(\mathrm{kpc})<1.7$ which is where both the X-ray and optical distance measurements overlap.

\section{Space velocity}

From the values of position, distance, proper motion and radial velocity, the velocity components $U_{\mathrm{LSR}}, V_{\mathrm{LSR}}$ and $W_{\mathrm{LSR}}$ relative to the Local Standard of Rest can be calculated using, for example, Johnson \& Soderblom's (1987) equations of transformation. Assuming that the Sun moves with components $\left(U_{\odot, \mathrm{LSR}}, V_{\odot, \mathrm{LSR}}, W_{\odot, \mathrm{LSR}}\right)=(10 ., 5.2,7.2) \mathrm{km} \mathrm{s}^{-1}$ (Dehnen \& Binney 1998) relative to the Local Standard of Rest, from the two extreme values of the distance we obtained: for $d=$ $0.9 \mathrm{kpc},\left(U_{\mathrm{LSR}}, V_{\mathrm{LSR}}, W_{\mathrm{LSR}}\right)=(204 \pm 21,-196 \pm 35,-122 \pm$ $38 \mathrm{~km} \mathrm{~s}^{-1}$, and for $d=1.7 \mathrm{kpc},\left(U_{\mathrm{LSR}}, V_{\mathrm{LSR}}, W_{\mathrm{LSR}}\right)=$ $(239 \pm 40,-303 \pm 67,-305 \pm 71) \mathrm{km} \mathrm{s}^{-1}$. This implies that the source moves towards the Galactic Centre and has a $3 \sigma$ vertical motion towards the Galactic plane. These two sets of values are rather different from the mean values of $U, V$ and $W$ that characterize the kinematics of stars belonging to the halo, and the thin and thick discs of the Galaxy (Chiba \& Beers 2000; Soubiran et al. 2003). The system velocities would be $v_{\mathrm{sys}}=308 \pm 51 \mathrm{~km} \mathrm{~s}^{-1}$ and $v_{\mathrm{sys}}=493 \pm 105 \mathrm{~km} \mathrm{~s}^{-1}$ for $d=0.9 \mathrm{kpc}$ and $d=1.7 \mathrm{kpc}$ respectively.

\section{Galactocentric orbit}

We integrated the orbit of Cen X-4 based on the heliocentric position and kinematic data listed in Table 3. In order to convert heliocentric positions and velocities into coordinates in the Galaxy frame we placed the Sun at $\left(X_{\odot}, Y_{\odot}, Z_{\odot}\right)=$ $(-8,0,0) \mathrm{kpc}$, with a velocity $\dot{\boldsymbol{r}}_{\odot} \equiv\left(U_{\odot}, V_{\odot}, W_{\odot}\right)=$ $(10.0,225.2,7.2)$ (Binney \& Merrifield 1998; Dehnen \& Binney 1998) and used the equation

$$
\begin{aligned}
\dot{\boldsymbol{r}}= & \dot{\boldsymbol{r}}_{\odot}+v_{\mathrm{r}}(\cos b \cos l, \cos b \sin l, \sin b) \\
& +r_{s} \mu_{l} \kappa(-\sin l, \cos l, 0) \\
& +r_{s} \mu_{b} \kappa(-\sin b \cos l,-\sin b \sin l, \cos b)
\end{aligned}
$$

where $r_{s}$ is the heliocentric distance, $\kappa \simeq 4.74$, a conversion factor from ( $\mathrm{kpc}$ mas/yr) to $\left(\mathrm{km} \mathrm{s}^{-1}\right), v_{\mathrm{r}}$, the heliocentric radial velocity and $\mu_{l}, \mu_{b}$, proper motions in the longitudinal and latitudinal direction, respectively.

We assumed a three-component Galaxy model that consists of: (i) a Miyamoto \& Nagai (1975) disc; (ii) a Hernquist bulge
(Hernquist 1990); and (iii) a spherical logarithmic halo. The Galaxy parameters can be found, for example, in Law et al. (2004).

Unfortunately, the heliocentric distance and velocity of Cen X-4 are poorly determined. Values with these large errors represent a large set of possible orbits that must be statistically treated. We calculated an initial set of 5000 orbits using Monte Carlo simulations, where the heliocentric distance is randomly chosen within the range given above. Proper motions were distributed following a Gaussian with variance equal to the measured error of $10 \mathrm{mas} / \mathrm{yr}$ and mean value $\left\langle\mu_{l}\right\rangle=-22 \mathrm{mas} / \mathrm{yr}$ and $\left\langle\mu_{b}\right\rangle=-53 \mathrm{mas} / \mathrm{yr}$. Subsequently, we integrated each orbit 1 Gyr backwards in time. In Fig. 2 we show the orbital parameters that result from the distribution of initial positions and velocities. The orbit of Cen X-4 appears totally inconsistent with typical orbits of Milky Way stars. This system has a perigalacticon of $r_{\mathrm{p}} \sim 1-4 \mathrm{kpc}$, whereas the apogalacticon occurs at $r_{\mathrm{a}} \sim 14-16 \mathrm{kpc}$ from the Galactic Centre. Although $r_{\mathrm{p}}$ and $r_{\mathrm{a}}$ appear to be fairly sensitive to the initial position and velocity, the resulting eccentricity, $e \equiv\left(r_{\mathrm{a}}-r_{\mathrm{p}}\right) /\left(r_{\mathrm{a}}+r_{\mathrm{p}}\right)=0.85 \pm 0.10$, barely depends on the errors from heliocentric distance and proper motion measurements. This eccentricity is considerably higher than that of disc stars $(e \simeq 0.5-0.1)$. The orbital inclination from the disc plane also shows a well defined distribution peak at $i \simeq 110^{\circ}$ and determines its orbital sense of motion to be retrograde for most of the distances considered in the distribution. This orbital inclination is considerably higher than for Milky Way stars (i.e. $i_{\odot}=4^{\circ}$ ) and leads to high vertical motions. As we can see in the upper right panel, Cen X-4 will move (or may have moved) in halo regions, with a most likely maximum vertical distance as large as $12-16 \mathrm{kpc}$.

In Fig. 3 we have represented the galactocentric orbits of Cen X-4 for two distances, 0.9 and $1.3 \mathrm{kpc}$. These orbits were calculated by integrating backwards in time for $460 \mathrm{Myr}$ and both orbits are highly eccentric. The orbit for $d=0.9 \mathrm{kpc}$ shows a prograde sense of motion, whereas for $d=1.3 \mathrm{kpc}$ the system describes a retrograde orbit. These orbits intersect the disc plane for the first time (integrating backwards in time) 148 and $243 \mathrm{Myr}$ ago for $d=0.9 \mathrm{kpc}$ and $d=1.3 \mathrm{kpc}$, respectively.

\section{Pinning down the formation region of Cen X-4}

Two main scenarios have been proposed to explain the current kinematic properties of LMXBs observed at high Galactic latitudes: (i) these binary systems originated in the Galactic bulge or in the disc and received a natal kick as a result of black hole/neutron star formation; or (ii) they formed in the Galactic halo either in dense globular clusters or as isolated ancient primordial binaries. Mirabel \& Rodrigues (2003) argued that the LMXB Sco X-1 most probably was formed in the core of a globular cluster in a close encounter of the compact object with a single star or with a binary (see Verbunt 2003, for a review).

Halo stars and globular cluster stars are generally metalpoor (with heavy elements between 10 and 1000 times less abundant than in the Sun). If the Cen X-4 system had originated in the halo, the secondary star would have a low 

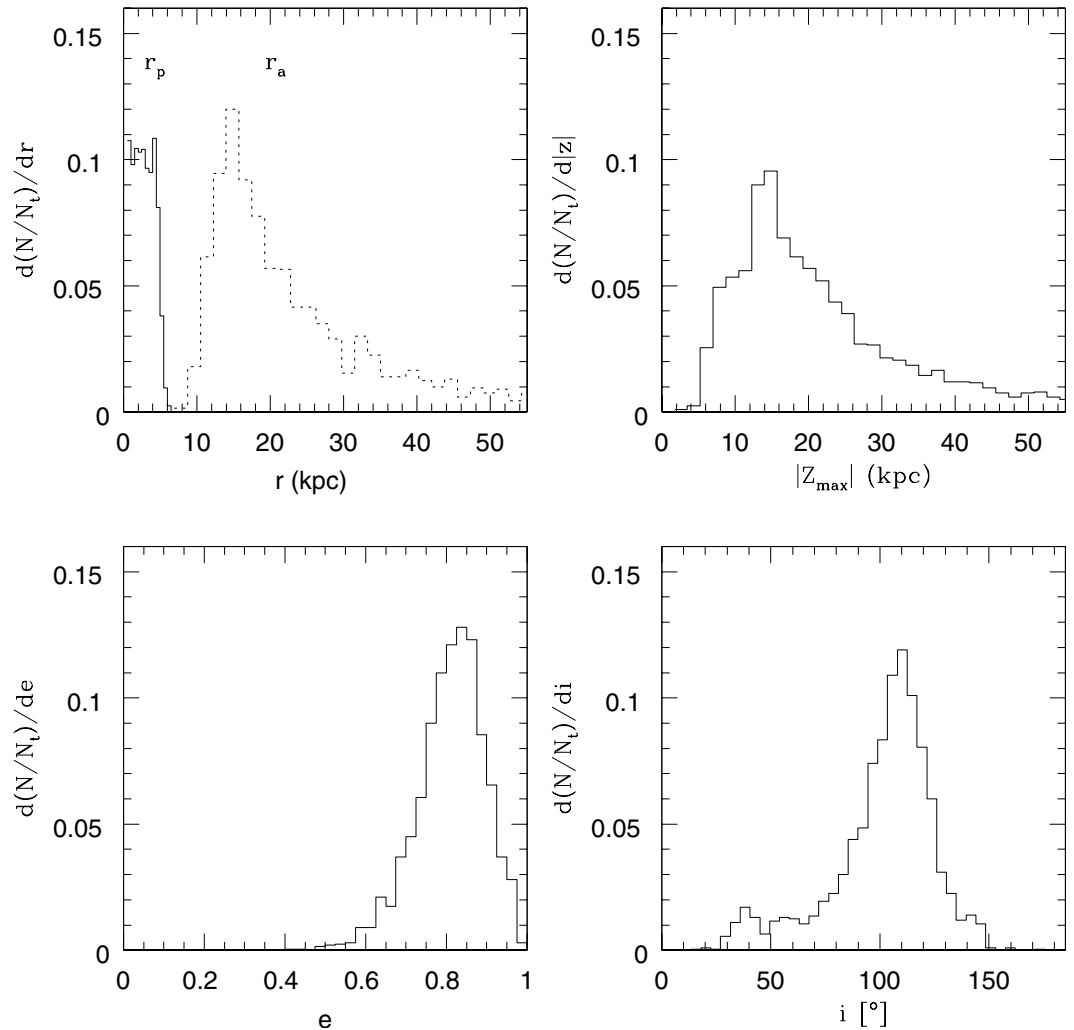

Fig. 2. Distribution of perigalacticon and apogalacticon distances (upper left panel), maximum vertical distance (upper right panel), eccentricities (lower left panel) and orbital inclinations (lower right panel). Distributions are normalized to the total number of orbits in our calculus $\left(N_{\mathrm{t}}=5000\right)$.
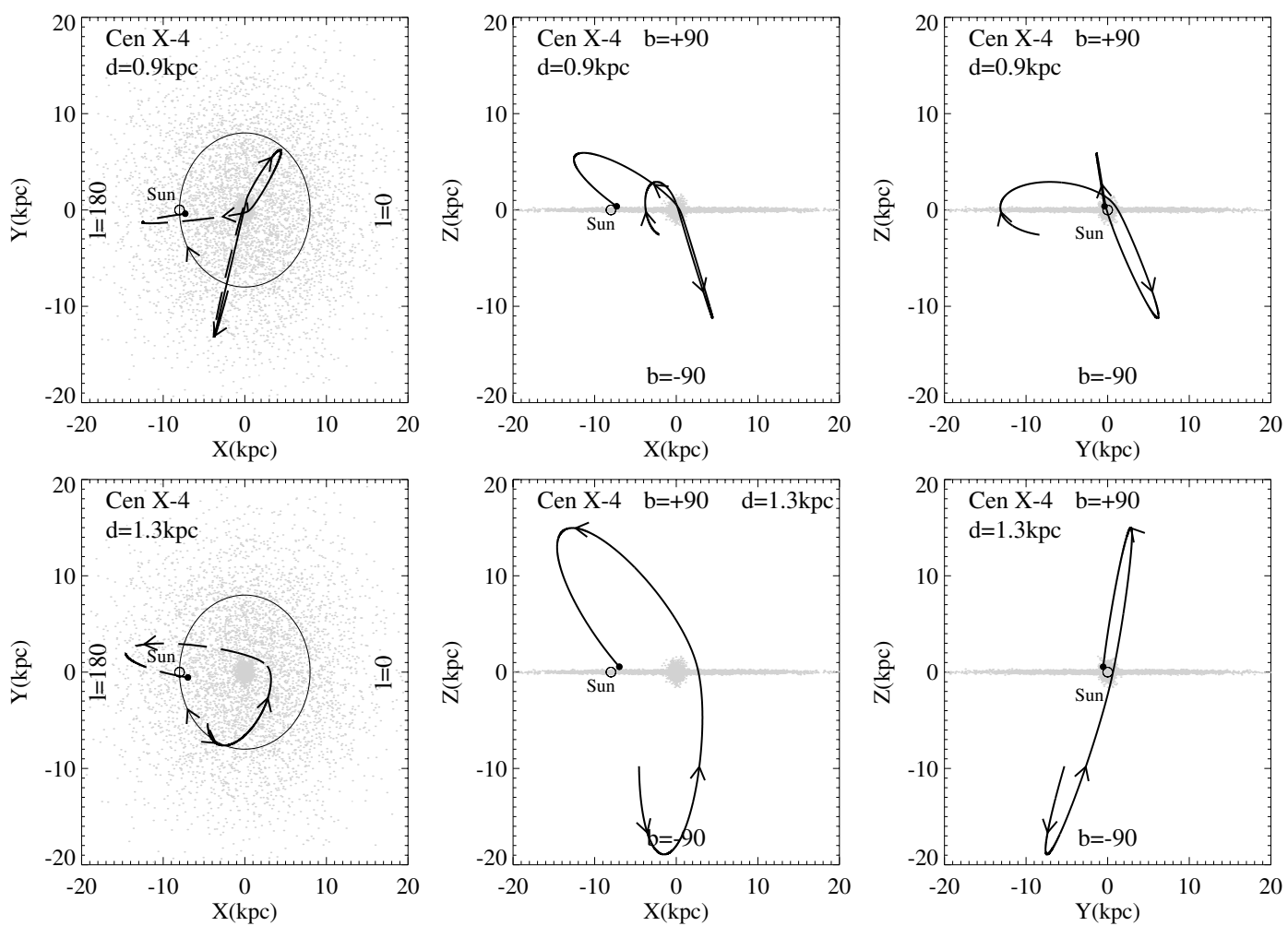

Fig. 3. Galactocentric orbits of Cen X-4 viewed from above the Galactic plane (left panels) and edge on (centre and right panels). The upper panels show the orbit for a heliocentric distance of $d=0.9 \mathrm{kpc}$ and the lower panels for $d=1.3 \mathrm{kpc}$. In the left panels, the solid line represents the section of the orbit in the Southern Galactic Hemisphere, whereas the dashed line shows the section of the orbit in the Northern Galactic Hemisphere. Both orbits were calculated by integrating backwards over $460 \mathrm{Myr}$. The circular orbit corresponds to the Sun. Open and filled circles show the current positions of the Sun and Cen X-4 respectively. 

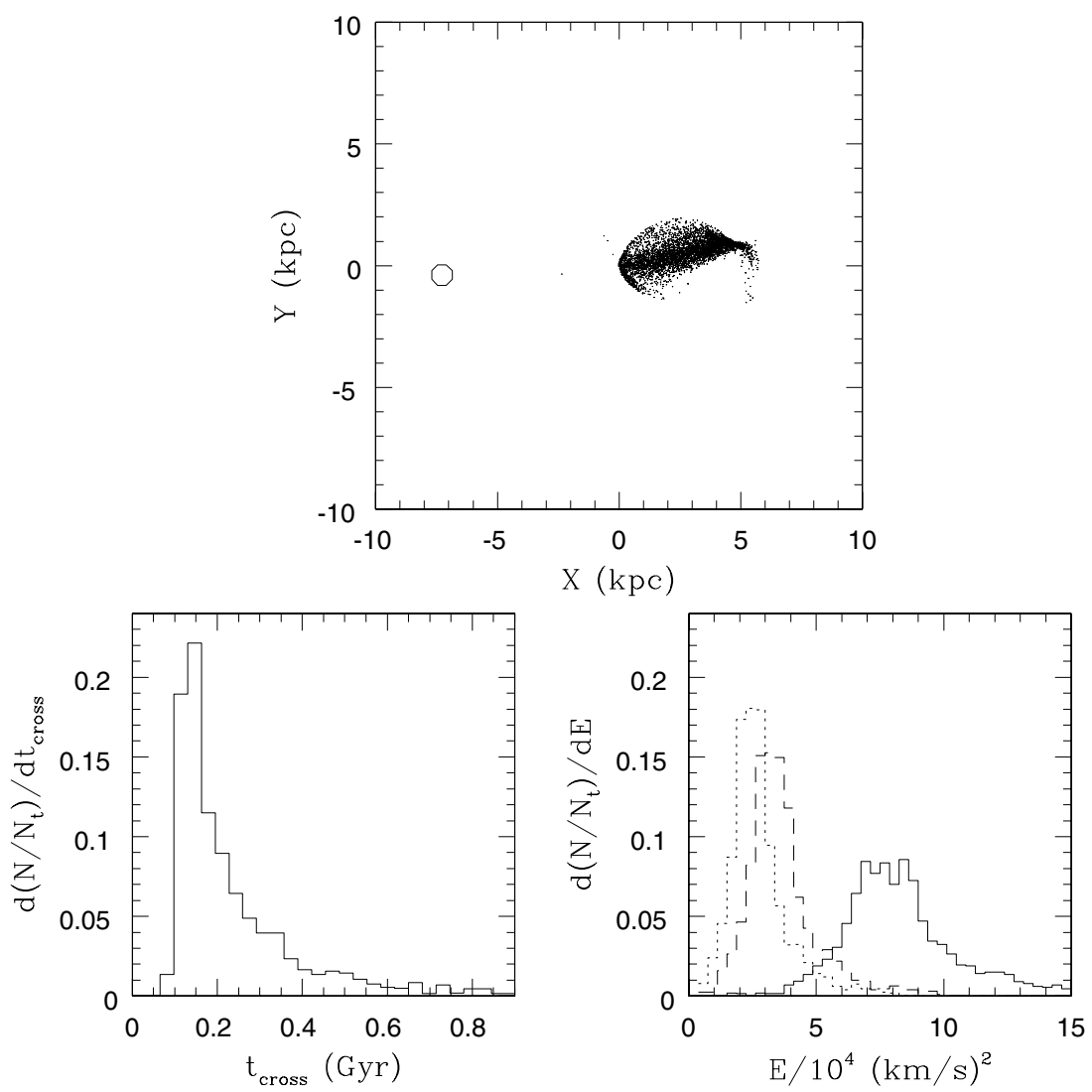

Fig. 4. Upper panel: first crossing of the axisymmetry plane when integrating the orbit of Cen X-4 backwards in time. The circle indicates its present position, and its diameter corresponds to the uncertainty in the distance estimate. Lower left panel: distribution of times when the orbit (integrated back in time) crosses the disc plane for the first time. Lower right panel: distribution of energies per unit of mass gained by Cen X-4 in comparison with circular orbits at the first-crossing position. The solid line represents the definition for the energy $E$ given in the text whereas the dotted and dashed lines show $E^{\prime}$ for $M_{\mathrm{He}}=4 M_{\odot}$ and $M_{2}=0.8 M_{\odot}$ for $M_{\mathrm{He}}=3 M_{\odot}$ and $M_{2}=0.5 M_{\odot}$ respectively.

metallicity. Recently, the iron content ${ }^{3}$ of the secondary star has been determined at roughly twice solar $([\mathrm{Fe} / \mathrm{H}]=0.23 \pm 0.1$, González Hernández et al. 2005). This high metal content, together with measured element abundance ratios in the companion star, makes it very unlikely that the system was formed in the Galactic halo, either in a globular cluster or as an ancient primordial binary. Most probably, the system formed in the Galactic disc and was expelled from its birth place as a consequence of a supernova (SN hereafter) explosion of the massive progenitor of the neutron star.

An origin in the Galactic disc requires that the system obtained enough energy to be expelled, most probably as a consequence of a natal kick in a SN explosion. In the lower right panel of Fig. 4 we show an estimate of the energy per unit of mass gained by the binary system due to a supernova at a first crossing. We define this energy as $E=E_{\text {orb }}-E_{\text {circ }}$, where $E_{\text {circ }}$ is the energy per unit of mass of a star following a circular orbit in the region where Cen X-4 crosses the Galactic plane. The resulting distribution is close to a Gaussian with $\langle E\rangle \simeq(8 \pm 1) \times 10^{4}\left(\mathrm{~km} \mathrm{~s}^{-1}\right)^{2}$. This energy must be lower than the difference between the total energy of the system after and before the SN explosion if the binary components remain bound. Brandt \& Podsiadlowski (1995) investigated

\footnotetext{
${ }^{3}[\mathrm{Fe} / \mathrm{H}]=\log [N(\mathrm{Fe}) / N(\mathrm{H})]_{\star}-\log [N(\mathrm{Fe}) / N(\mathrm{H})]_{\odot}$.
}

the effects of high SN kick velocities on the orbital parameters of post-SN neutron star binaries. Using their formalism we compared the pre-SN, $E_{\text {pre-SN }}$, and post-SN, $E_{\text {post-SN }}$, total energies per unit of mass of the system after undergoing $\mathrm{SN}$ explosions. We defined this energy balance as $E^{\prime}=$ $E_{\text {post-SN }}\left(M_{\mathrm{T}, \text { post-SN }} / M_{\mathrm{T} \text {,pre-SN }}\right)-E_{\text {pre-SN }}$ where $M_{\mathrm{T} \text {,post-SN }}$ and $M_{\text {T,pre-SN }}$ are the total masses of the system after and before the $\mathrm{SN}$ event respectively. We found that energy released in a SN of He core progenitor of mass $M_{\mathrm{He}}=4 M_{\odot}$ and a secondary star of $M_{2}=0.8 M_{\odot}$ is $E^{\prime} \sim 1-3 \times 10^{4}\left(\mathrm{kms}^{-1}\right)^{2}$, whereas for $M_{\mathrm{He}}=3$ $M_{\odot}$ and $M_{2}=0.5 M_{\odot}, E^{\prime} \sim 1-2 \times 10^{4}\left(\mathrm{~km} \mathrm{~s}^{-1}\right)^{2}$. These energies are consistent with the energy required to launch Cen X-4 into an orbit like this from a birth place in the Galactic disc (see Fig. 4). A detailed study of the distribution of the kick directions with respect to the orbital motions of each component shows that it strongly depends on the masses of the different components of the system before and after the SN explosion. Thus the kick direction cannot randomly be chosen and is more restricted when the differences between the masses of both components are higher.

The abundance of lithium in the secondary star of Cen X-4 $\left(\log \epsilon(\mathrm{Li})_{\mathrm{LTE}}=3.06 \pm 0.29\right)$ is close to the cosmic Li abundance in the Galactic disc (Martín et al. 1994a; González Hernández et al. 2005) and thus substantially higher than in stars of comparable mass of the young Pleiades cluster whose 
age is $~ 120$ Myr (Martín et al. 1998; Stauffer et al. 1998). This might indicate that the system is relatively young since $\mathrm{Li}$ is severely depleted in the atmospheres of such stars during pre-main sequence and main sequence evolution. In order to explore the possibility of a recent formation of Cen X-4 in the Galactic disc, we calculated the positions where the orbit intersects the Galactic plane for the first time (integrating backwards in time). The result is plotted in the upper panel of Fig. 4, which shows that the orbit crosses the inner region of the Milky Way in the range $0-4 \mathrm{kpc}$ from the Galactic Centre. The distribution of times (lower left panel) indicates that the system was probably in the Galactic disc $\sim 100-200 \mathrm{Myr}$ ago. The high Li abundance of the secondary star might be explained if the system had formed at that moment. However, it has been proposed that a Li production mechanism via spallation reactions could be responsible for the high Li abundances of LMXBs (Martín et al. 1994b; Yi \& Narayan 1997; Guessoum \& Kazanas 1999). No direct evidence for such a mechanism has been found yet in Cen X-4 or in any other LMXB. Nevertheless, we cannot rule out that such a mechanism has been active in Cen X-4 and, thus, that the system could be much older.

\section{Conclusions}

We have used optical relative astrometry of the secondary star of the neutron star binary Cen X-4 to measure its proper motion. This proper motion, together with the estimated distance and radial velocity and its celestial coordinates, allows us to determine the space velocity components. These appear to be rather different from the mean values of $U, V$ and $W$ for stars belonging to the halo, the thin and the thick disc of the Galaxy. The system is falling into the Galactic plane with a large vertical velocity $\gtrsim 100 \mathrm{~km} \mathrm{~s}^{-1}$.

The high metallicity $([\mathrm{Fe} / \mathrm{H}]=0.23 \pm 0.1$, González Hernández et al. 2005) and the element abundance ratios of the companion star in Cen X-4 makes it very unlikely that the system formed in the Galactic halo. Most probably, the system originated in the Galactic disc and was propelled into its current position by a natal kick in a supernova explosion.

We have explored the galactocentric orbit of Cen X-4 through a statistical analysis that takes into account the uncertainty in the proper motion and the distance. The orbital inclination from the Galactic plane is $\simeq 110^{\circ}$ and determines its orbital sense of motion to be retrograde in a highly eccentric $(e \simeq 0.85 \pm 0.1)$ orbit. Following this orbit backwards, we have found that the system was probably at the Galactic disc 100-200 Myr ago. The formation of the neutron star at that time would imply a relatively young system and may provide a natural explanation for the observed high $\mathrm{Li}$ abundance of the secondary star. However, if there is an active Li production mechanism in Cen X-4, the Li abundance cannot be directly interpreted as an indication of youth, and it cannot be discarded that the system formed at much earlier times.
Acknowledgements. This work has made use of IRAF facilities and has been partially financed by the Spanish Ministry projects AYA2001-1657 and AYA2002-03570.

\section{References}

Belian, R. D., Conner, J. P., \& Evans, W. D. 1972, ApJ, 171, L87

Bessell, M. S., Castelli, F., \& Plez, B. 1998, A\&A, 333, 231

Binney, J., \& Merrifield, M., 1998, Galactic Astronomy (Princeton, New Jersey: Princeton University Press)

Blair, W. P., Raymond, J. C., Dupree, A. K., et al. 1984, ApJ, 278, 270

Brandt, N., \& Podsiadlowski, P. 1995, MNRAS, 274, 461

Chevalier C., Ilovaisky, S. A., van Paradijs, J., Pedersen, H., \& van der Klis, M. 1989, A\&A, 210, 114

Chiba, M., \& Beers, T. C. 2000, AJ, 119, 2843

Conner, J. P., Evans, W. D., \& Belian, R. D. 1969, ApJ, 157, 157

Cowley, A. P., Hutchings, J. B., Schmidtke, P. C., et al. AJ, 95, 1231

Dehnen, W., \& Binney, J. J. 1998, MNRAS, 298, 387

Gessoum, N., \& Kazanas, D. 1999, ApJ, 512, 332

Gualandris, A., Colpi, M., Portegies Zwart, S., \& Possenti, A. 2005, ApJ, 618, 845

González Hernández, J. I., Rebolo, R., Israelian, G., et al. 2005, submitted

Hernquist, L. 1990, ApJ, 356, 359

Jonker, P. G., \& Nelemans, G. 2004, MNRAS, 354, 355

Johnson, D. R. H., \& Soderblom, D. R. 1987, AJ, 93, 864

Law, D. R., Johnston, K. V., \& Majewski, S. R. 2004 [arXiv:astro-ph/0407566]

Lyne, A. G., \& Lorimer, D. R. 1994, Nature, 369, 127

Martín, E. L., Rebolo, R., Casares, J., \& Charles, P. A. 1994a, ApJ, 435, 791

Martín, E. L., Spruit, H. C., \& van Paradijs, J. 1994b, A\&A, 291, L43

Martín, E. L., Basri, G., Gallegos, J. E., et al. 1998, ApJ, 499, 61

Matsuoka, M., Inoue, H., Koyama, K., et al. 1980, ApJ, 240, 137

Mirabel, I. F., \& Rodrigues, I. 2003, A\&A, 398, L25

Mirabel, I. F., Dhawan, V., Mignani, R. P., Rodrigues, I., \& Guglielmetti, F. 2001, Nature, 413, 139

Miyamoto, M., \& Nagai, R. 1975, PASJ, 27, 533

van Paradijs, J., Verbunt, F., van der Linden, T., Pedersen, H., \& Wamsteker, W. 1980, ApJ, 241, 161

Schaifers, K., Voigt, H. H., Landolt, H., Börnstein, R., \& Hellwege, K. H. 1982, Landolt-Börnstein, Astronomy and Astrophysics. C: Interstellar Matter, Galaxy, Universe, Numerical Data and functional Relationships in Science and Technology, New Series (Berlin, Heildelgerg, New york: Springer-Verlag)

Shahbaz, T., Naylor, T., \& Charles, P. A. 1993, MNRAS, 265, 655

Soubiran, C., Bienaymé, O., \& Siebert, A. 2003, A\&A, 398, 141

Stauffer, J. R., Schultz, G., \& Kirkpatrick, J. D. 1998, ApJ, 499, 199

Torres, M. A. P., Casares, J., Martínez-Pais, \& Charles, P. A. 2002, MNRAS, 334, 233

Verbunt, F. 2003, New Horizons in Globular Cluster Astronomy, ed. G. Piotto, G. Meylan, S. George Djorgovski \& M. Riello, Padova, Italy, ASP Conference Proceedings, 296, 245

[arXiv:astro-ph/0210057]

Yi, I., \& Narayan, R. 1997, ApJ, 486, 363 